Article

\title{
Overexpression of PLIN1 Promotes Lipid Metabolism in Bovine Adipocytes
}

\author{
Shijun Li ${ }^{1,+}$, Sayed Haidar Abbas Raza ${ }^{1,+}{ }^{\oplus}$, Chunping Zhao ${ }^{1}$, Gong Cheng ${ }^{1}$ and \\ Linsen Zan 1,2,* \\ 1 College of Animal Science and Technology, Northwest A\&F University, Yangling 712100, China; \\ lishijun1990cn@163.com (S.L.); haiderraza110@nwafu.edu.cn (S.H.A.R.); zhao.chunping@foxmail.com (C.Z.); \\ chenggong@nwafu.edu.cn (G.C.) \\ 2 National Beef Cattle Improvement Center, Northwest A\&F University, Yangling 712100, China \\ * Correspondence: zanlinsen@163.com; Tel.: +86-29-8709-1923; Fax: +86-29-8709-2164 \\ + These authors contributed equally to this work.
}

Received: 3 September 2020; Accepted: 20 October 2020; Published: 22 October 2020

Simple Summary: In bovine preadipocytes, the overexpression of the PLIN1 gene promotes the expression of FASN, PPAR $\gamma, A C C, L P L, F A B P 4, D G A T 2$, and C/EBP $\beta$ at the mRNA level, inhibiting the expression of fat metabolism genes such as PLIN2 and ATGL. Furthermore, after overexpression of PLIN1, the oil red O-staining results showed that the number of lipid droplets (LDs) in fat cells, their volume and triglyceride content were increased. The elevation in triglyceride content indicates that PLIN1 can promote the accumulation of triglyceride in bovine preadipocytes and has an important regulatory role in fat metabolism. After transfection with the adenovirues Ad-PLIN1 and Ad-NC (empty virus), RNA-seq was used to analyze the differentially expressed genes in bovine preadipocytes. A total of 1923 differentially expressed genes were detected, and GO and KEGG signaling pathways were analyzed for differentially expressed genes.

Abstract: Perilipin 1 (PLIN1) is a protein encoded by the PLIN1 gene in eukaryotes. PLIN1 is a member of the PAT protein family, a family of proteins related to lipid droplet (LD) surface proteins. PLIN1 phosphorylation plays a vital role during fat metabolism of adipose tissue lipolysis and fat storage in adipocytes. However, to further explore the regulation of the PLIN1 gene on the proliferation, differentiation and lipid metabolism of bovine adipocytes. In this study, the mRNA expression of PLIN1, at day six, was the highest during bovine adipocyte differentiation. Moreover, PLIN1 can promote the proliferation and differentiation of preadipocytes in cattle. On the sixth day, after transfection with, and overexpression of, the PLIN1 gene in bovine preadipocytes via adenovirus, cell samples were collected, and transcriptome sequencing was performed. A total of 1923 differentially expressed genes were detected. Through GO and KEGG pathway analysis, the differentially expressed genes were established to be mainly enriched in the AMPK, Wnt, and PPAR signaling pathways related to fat proliferation and differentiation. In conclusion, at the transcriptional level, PLIN1 plays an important role in regulating fat proliferation and metabolism. Additionally, the sequencing results screened new differentially expressed genes related to fat metabolism, providing theoretical support for molecular breeding of Qinchuan beef cattle.

Keywords: cattle; PLIN1 gene; bovine adipocytes; adenovirus; RNA-seq; differentially expressed genes

\section{Introduction}

For beef cattle, the marbling grade of meat is an important criterion reflecting the quality of beef [1], and marbling is the major determinant of consumer acceptability [2]. Qinchuan cattle is an 
indigenous Chinese breed and one of the five finest yellow cattle in China. It is mainly produced in the Guanzhong Plain of Shaanxi. This variety has the advantage of fast growth rate, but the meat quality traits, such as intramuscular fat content are still not comparable with the exotic varieties like Wagyu. The marble pattern of beef is mainly affected by intramuscular fat content [3]. In mammals, fat is predominantly stored as subcutaneous, visceral, or intramuscular fat. Intramuscular fat content is an important factor in determining meat quality [4,5]. Fat deposition in muscles involves a series of processes, such as preadipocyte proliferation, preadipocyte differentiation, and lipid metabolism, and is regulated by related functional genes [6]. Therefore, it is particularly important to study the mechanism of fat formation and the regulation of genes related to meat quality traits at the molecular level. Lipid droplets (LDs) are common dynamic cellular organelles that contain unique surface proteins in most mammalian cells and are present in various cell types. The PAT family of proteins not only play pivotal roles in the formation and degradation of lipid droplets, but also regulates TG (triacylglycerol) packaging. The PAT family is comprised of five different lipid droplet proteins in mammals: Perilipin, adipose differentiation-related protein (ADRP), tail-interacting protein of 47 kilo Daltons (TIP47), S3-12, and OXPAT [7]. The PAT family proteins play important roles in regulating intracellular lipid storage, and are widely present in many species [8]. All PAT proteins exhibit sequence similarity and possess the ability to bind intracellular LDs. The differences between various PAT proteins affect their functions in cells. However, all PAT proteins may perform the function of regulating LDs within the cellular environment [9].

The adenovirus AdEasy system is a simple and convenient adenovirus recombination system composed of an adenovirus shuttle vector (pAdTrack or pShuttle) and a backbone vector (pAdEasy), which was constructed by He in 1998 [10]. Firstly, a recombinant adenovirus plasmid can be produced by inserting a target gene into a shuttle vector (e.g., pAdTrack-CMV) and then inserting a recombinant adenovirus. After transfection of these plasmids into a mammalian packaging cell line, virus production is conveniently carried out through green fluorescent protein. The overexpression of, and interference with, target genes to detect their effects at the cellular, mRNA, and protein levels are important methods for studying gene function. Adenovirus vectors are currently the most effective transgenic vectors, and they have the advantages of high infection efficiency, low pathogenicity, and high titer [11]. Adenovirus vectors can be used in cells of different periods and are widely employed in human gene therapy [12]. The effect of the PLIN1 gene on lipid metabolism in bovine preadipocytes is rarely reported. PLIN1 is one of the most abundant protein on LDs in the adipocytes. The perilipin 1 protein coats the outer layer of LDs to prevent lipases in the body, such as hormone-sensitive lipase (HSL) and lipase activator (ATGL), from entering LDs [13]. In the adipose tissue of PLIN1-null mice, some sterol biosynthetic enzymes were downregulated [14], indicateing that PLIN1 deficiency may affect the sterol biosynthetic pathway in steroidogenic cells as PLIN1 activates HSL. Lipolysis in the adipose tissue was significantly reduced, and the adipose tissue was enhanced in PLIN1-null mice [15]. Studies have compared different levels of lipolysis in PLIN1-null and wild-type mice and found that PLIN1-null adipocytes had reduced catecholamine-stimulated lipolysis and increased rates of constitutive (unstimulated) lipolysis [16]. PLIN1 is essential during the liposecretion process, and it can be expressed on the LD surfaces in white and brown mature adipocytes [17]. Studies have shown that PLIN1 is an important regulator of lipolysis and protects LDs from basal lipolysis. However, when fat cells are stimulated to initiate lipolysis, PLIN1 regulates lipohydrolase in LDs to complete the hydrolysis [18]. Accordingly, PLIN1 knockout mice are lean and protected from diet-induced obesity [19]. Importantly, the absence of PLIN1 causes dysregulation of adipogenic signaling, impairing the development and differentiation of adipose cells [20]. In PLIN1-/- mice, both adipogenic transcription factors, such as PPAR $\gamma$ and C/EBPs, and lipolysis-related enzymes, such as HSL and ACC1, were downregulated [21]. Previous studies have revealed that PLIN1 plays a key role in adipogenesis and LD formation, and that PLIN1 may participate in the process of the liposecretion process of mature adipocytes [16,22]. Proliferation and liposecretion are regulated through key pathways, including Akt, Erk, and Wnt pathways [21]. In particular, TGF $\beta$ is mediated through various PLIN1 proteins which have specific activities either as differentiation 
or proliferation modulators [23]. However, the molecular mechanisms involved in the regulation of lipid metabolism by PLIN1 remain unclear in bovine adipocytes. The PLIN1 gene in Qinchuan cattle may feature prominently in fat proliferation and differentiation. Therefore, this study investigated the differentially expressed genes (DEGs) and related signaling pathways through transcriptome sequencing of cell samples. In addition, we studied the functional network of LD formation and fat metabolism, and speculated on the potential pathways that regulate fat metabolism. These findings have improved our understanding of lipid metabolism and provide theoretical support for molecular breeding of Qinchuan beef cattle in Qinchuan.

\section{Materials and Methods}

\subsection{Sample Information}

The animal samples for the experiments were approved in accordance with code NWAFUC/110 of Northwest A\&F University, China, and the use of experimental animals followed all guidelines of the organization and government.

\subsection{Construction of Interference and Overexpression Adenovirus Vectors of PLIN1}

An overexpression adenovirus vector, with a full-length bovine PLIN1 gene and coding sequence, was developed as previously described and termed as Ad-PLIN1 [23]. Firstly, the full-length coding sequence of the PLIN1 gene was amplified and then, without the site mutation sequence, the pAd-Track shuttle vector was selected and cloned. The sequence of PLIN1 was cloned into the pAd-Track shuttle vector. The shutter vector was linearized by Pme I restriction enzyme (NEB, USA) and recombined with the pAdEasy-1 vector in BJ5183 competent cells to achieve the homologous recombination of shuttle vector and skeleton vector in cells, and the adenovirus recombinant pAd-PLIN1 was obtained. Finally, the pAd-PLIN1 expression vector was removed by the extraction of gel after it was digested with the Pac I restriction enzyme (NEB, USA) and converted into HEK 293A cells using the liposome method to be packed into an adenovirus (Ad-PLIN1). The National Beef Cattle Development Center has retained and developed negative control of adenovirus (Ad-NC).

To construct an interference adenovirus vector for negative control oligonucleotides (sh-NC) and unique short-hairpin RNA (shRNA) for bovine PLIN1 mRNA, this study used online application called BLOCK-iT Designer RNAi (Thermo fisher scientific, Waltham, MA, USA). Subsequently, we cloned shRNAs into the pENTR/U6 RNAi input vector and the psiCHECK-II reporter device (Promega, Madison, WI, USA) to detect interference efficiencies of the pENTR/U6 RNAi input vector. shRNA-1337 (Table 1) was chosen with a maximum intervention quality of 76.2 percent, which is acceptable for adenovirus packaging. The pENTRTM/U6-shRNA-1337 was then recombined with the $\mathrm{pAd} / \mathrm{PL}-\mathrm{DEST} \mathrm{T}^{\mathrm{TM}}$ disruption vector. Pursuant to LR Clonase Control II Enzyme (Invitrogen, Carlsbad, CA, USA), transmitted vector (Invitrogen, Carlsbad, CA, USA) in vitro. Adenovirus plasmid recombinant sh-PLIN1 was obtained and transferred to HEK 293A cells to enable adenovirus (sh-PLIN1) to be packaged.

Table 1. Exact shRNA and sh-NC sequences for the PLIN1 mRNA.

\begin{tabular}{ccccc}
\hline $\begin{array}{c}\text { Name of } \\
\text { shRNAs }\end{array}$ & Sense Strand $\left(\mathbf{5}^{\prime}-\mathbf{3}^{\prime}\right)$ & Loop & Anti-Sense Strand $\left(\mathbf{5}^{\prime} \mathbf{- 3}^{\prime}\right)$ & $\begin{array}{c}\text { RNA Poly III } \\
\text { Terminator }\end{array}$ \\
\hline shRNA-264 & GCCTCTGTATGCAATGCCTAC & TCAAGAG & GTAGGCATTGCATACAGAGGC & TTTTTT \\
shRNA-786 & GCCAACACTCTCTCTAGACAC & TCAAGAG & GTGTCTAGAGAGAGTGTTGGC & TTTTT \\
shRNA-1337 & GATGGAACCCGAGAGCGAATT & TCAAGAG & GAATTCGCTCTCGGGTTCCATC & TTTTTT \\
shRNA-NC & GTTCCACGACCAAATCAGCTC & TCAAGAG & GAGCTGATTTGGTCGTGGAAC & TTTTTT \\
\hline
\end{tabular}

\subsection{Isolation, Culture, and Differentiation of Bovine Preadipocytes}

Bovine preadipocytes were collected from five-day-old healthy Qinchuan calves according to the method described in the previous literature [24,25]. First, the fat tissue near the longissimus dorsi 
muscle was taken from healthy calves under aseptic conditions. The fat tissue was first washed with $75 \%$ ethanol, cut into small tissue pieces, and transferred into a $15 \mathrm{~mL}$ centrifuge tube. The sample was washed with $1 \times$ PBS three times and immediately transferred to the cell culture room. The cells were inoculated into T75 culture flasks at a density of $5 \times 10^{4} \mathrm{cells} / \mathrm{cm}^{2}$, and cultured in an incubator (Thermo Scientific, Waltham, MA, USA) at $37^{\circ} \mathrm{C}$ in saturated water with $5 \% \mathrm{CO}_{2}$. After centrifugation, the preadipocytes were inoculated in an appropriate amount of complete Dulbecco's modified Eagle medium (DMEM/F12) (Gibco, Shanghai, China). After being cultured until contact inhibition for $2 \mathrm{~d}$, the induction solution was replaced with DMEM/F-12 supplemented with 10\% FBS (PAN-Biotech, USA), 1\% penicillin/streptomycin, 0.5 mM IBMX (Sigma, St. Louis, MO, USA), $1 \mu$ M DEX (Sigma, St. Louis, MO, USA), and $5 \mu \mathrm{g} / \mathrm{mL}$ bovine insulin (Sigma, St. Louis, MO, USA) to induce differentiation. The appearance of lipid droplets can be observed by the inverted fluorescence microscope (Olympus IX71, Tokyo, Japan).

\subsection{Tissue Collection and Quantitative Reverse-Transcription PCR (RT-PCR) Analysis}

For tissue collection, 12 tissue samples were obtained from 3 adult male Chinese indigenous Qinchuan cattle, including- subcutaneous fat, longissimus thoracis, and kidney. Total RNA was extracted from the tissue using TRIzol ${ }^{\mathrm{TM}}$ Reagent (Invitrogen, Carlsbad, CA, USA) and then cDNA libraries were constructed using Prime Script ${ }^{\mathrm{TM}} \mathrm{RT}$ reagent kit (Takara, China). RT-PCR was performed following the protocol of the SYBR Green PCR Master Mix kit (Takara, Beijing, China) and 7500 System (Applied Biosystems, Foster City, CA, USA). The expression levels of target mRNA were calculated using the $2^{-\Delta \Delta \mathrm{Ct}}$ method [26].

\subsection{Western Blotting}

Total protein was extracted from bovine adipocytes using a lysis buffer with protease inhibitors. Protein samples were added to a 12\% SDS-PAGE gel at $20 \mu \mathrm{g}$ per well for electrophoretic migration; thereafter, the protein bands on the SDS-PAGE gel were transferred to a polyvinylidene difluoride (PVDF) membrane. The membrane was then incubated with a primary antibody was incubated overnight at $4{ }^{\circ} \mathrm{C}$. The membrane was then washed with phosphate buffer (PBST) for three times and incubated with a secondary antibody for $2 \mathrm{~h}$ at room temperature. The membrane was then exposed to detect luminescence signals using a ChemiDoc. XRS+ System (Bio-Rad, Hercules, CA, USA).

\subsection{Oil Red O Staining and Cellular Triglyceride Determination}

Cellular triglyceride levels were determined as previously described $[27,28]$. Bovine preadipocytes were inoculated into six-well cell culture plates. After $6 \mathrm{~d}$ of induction of differentiation, the cells were collected by trypsinization, centrifugation and then lysed. A total of $0.1 \mathrm{~mL}$ of the lysate could be added per $1 \times 10^{6}$ cells proportionally. Standard samples were prepared using $4 \mathrm{mM}$ glycerol standards and distilled water. The triglycerides cleaved from the cells were extracted with a tissue triglyceride assay kit (Applygen Technologies Inc., Beijing, China), and the OD value was then measured at $550 \mathrm{~nm}$ using a microplate reader. Finally, the concentration of triglyceride was calculated from a standard curve prepared from the OD value of the standard sample. Triglyceride levels were normalized to the protein concentration of each sample. Finally, the concentration of triglycerides was calculated from a standard curve prepared from the OD value of the standard sample. After 6 days of differentiation induction, the culture medium of the cells was discarded. Thereafter, the cells were washed three times with phosphate-buffered saline (PBS), fixed with $4 \%$ paraformaldehyde for $30 \mathrm{~min}$. Oil red O working solution was added to cover the bottom of the cell culture dish, and the cells were stained for $30 \mathrm{~min}$ at room temperature. The cells images were captured using an Olympus $1 \times 71$ microscope (Olympus IX71, Tokyo, Japan). 


\subsection{The 5-ethynyl-2-deoxyuridine (EdU) Proliferation Assay}

EdU assay was performed using the cell light EdU DNA proliferation kit (RiboBio, Suzhou, China). Bovine preadipocytes were seeded in 24-well cell culture plates. When the cell density reached 50-60\% density, the cells were transfected with Ad-NC, Ad-PLIN1, sh-NC, or sh-PLIN1. After 24 h, the cells were incubated with EdU medium for $2 \mathrm{~h}$. The EdU assay was performed according to the manufacturer's protocol. The cell images were captured using an Olympus microscope (Olympus IX71, Tokyo, Japan).

\subsection{Cell Cycle Assay through Flow Cytometry}

Bovine preadipocytes were seeded in six-well cell culture plates, and the cells were transfected with Ad-NC, Ad-PLIN1, sh-NC, or sh-PLIN1. The cells were collected, washed with 1XPBS, and resuspended in $1 \times$ PBS. The cell cycle was analyzed through flow cytometry (FACS CantoTM II, BD BioSciences, San Jose, CA, USA) by counting 20,000 cells.

\subsection{RNA Sequencing (RNA-seq)}

The bovine preadipocytes were cultured in $10 \mathrm{~cm}$ cell culture plates. Cell samples were collected after induction of differentiation at $6 \mathrm{~d}$. RNAs from three biological replicates of each group were isolated using RNeasy kit (Qiagen China (Shanghai) Co Ltd., Shanghai, China). The cell samples were subsequently sent to Novo Genefor transcriptome sequencing.

\subsection{Identification of DEGs and Functional Enrichment Analysis}

The edgeR package (http://www.r-project.org/) was used to identify DEGs within samples. GO term enrichment analysis was performed using the online Visualization and Integrated Discovery (DAVID) software [29,30] for the three GO categories. In addition, KEGG pathway enrichment analysis was carried out using the KEGG Orthology-Based Annotation System (KOBAS) software [31,32]. Significant enrichment was considered for a corrected $p$-value $<0.05$.

\subsection{1. qRT-PCR Validation of Key Genes}

Total RNA from bovine adipocytes cells was extracted using RNAiso (Takara, Beijing, China). A SYBR ${ }^{\circledR}$ Premix Ex Taq ${ }^{\mathrm{TM}}$ II Kit (Takara) was used to carry out qRT-PCR with an ABI 7500 Real-Time PCR system (Applied Biosystems, Foster City, CA, USA). GAPDH served as an internal reference to normalize gene expression levels via the $2^{-\Delta \Delta C t}$ method [33].

\subsection{Statistical Analyses}

The data were expressed as the mean \pm standard deviation and analyzed through analysis of variance (ANOVA) using the Statistical Package for the Social Sciences (SPSS), version 17(IBM, Armonk, NY, USA). The results are shown as mean \pm standard error of the mean (SEM).

\section{Results}

\subsection{Expression of PLIN1 in Bovine Preadipocytes}

To elucidate the function of the PLIN1 gene in different tissues of Qinchuan cattle, the expression levels of PLIN1 were determined using qRT-PCR. The expression of PLIN1 was highest in subcutaneous fat and lowest in the kidney (Figure 1A; $p<0.05$ ). In order to systematically research the temporal expression of PLIN1 during bovine preadipocyte differentiation, this study built the differentiation pattern of small adipocytes, culture the cells within $9 \mathrm{~d}$, and detect the expression level of PLIN1 every $3 \mathrm{~d}$. The adenoviruses Ad-NC, Ad-PLIN1, sh-NC, and sh-PLIN1 were transfected into bovine preadipocytes. The total RNA of cells at $0 \mathrm{~d}, 3 \mathrm{~d}, 6 \mathrm{~d}$, and $9 \mathrm{~d}$ was collected. After interfering with the PLIN1 gene, the mRNA expression of PLIN1 was reducing (Figure 1B; $p<0.05$ ). Moreover, after 
overexpression of PLIN1, the mRNA expression of PLIN1 increased with an increase in induction differentiation time, and the expression of $6 \mathrm{~d}$ was the highest (Figure $1 \mathrm{C} ; p<0.05$ ).

A

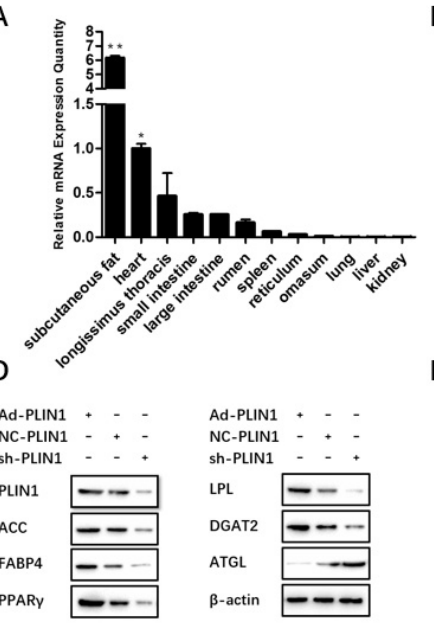

B

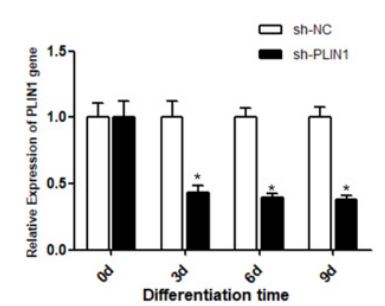

E

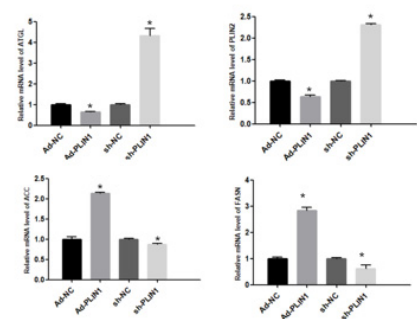

C
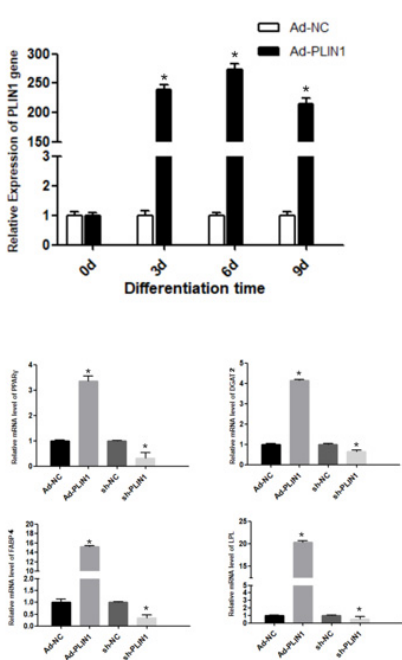

Figure 1. Expression patterns of the PLIN1 gene during bovine preadipocyte differentiation. (A) The relative expression of the PLIN1 gene was detected by qPCR in12 different bovine tissues. (B) After infecting adipocytes with sh-PLIN1 and sh-NC, the expression of PLIN1 gene decreased in bovine preadipocytes. (C) After allowingoverexpression of Ad-PLIN1 and Ad-NC in adipocytes, the expression of PLIN1 decreased in bovine preadipocytes. (D) Effects of overexpression and interference of the PLIN1 gene on the protein expression levels of lipid metabolism-related genes. (E) Effects of overexpression of and interference with PLIN1 on mRNA expression level of fat-related genes. The values represent mean \pm SEM $(\mathrm{n}=3) .{ }^{*} p<0.05$.

Furthermore, the expression levels of PLIN1, adipose differentiation marker genes, and lipid metabolism marker genes were detected on the sixth day. The protein expression levels of PLIN1, ACC, PPAR $\gamma, D G A T 2, F A B P 4, A T G L$, and LPL were detected (Figure 1D; $p<0.05$ ). The protein expression levels of PLIN1 were significantly up-regulated when treated with Ad-PLIN1 and decreased significantly when sh-PLIN1 was used (Figure 1D; $p<0.05$ ). To explore the effect of PLIN1 on lipid metabolism, the expression levels of genes related to lipid metabolism in adipocytes were determined. After overexpression of PLIN1, the mRNA expression levels of ACC, FASN, PPAR $\gamma, D G A T 2, F A B P 4$, and $L P L$ were significantly increased, and the protein levels of FAS and PPAR $\gamma$ also increased. On the contrary, after interfering with the PLIN1 gene, ACC, FASN, PPAR $\gamma, D G A T 2, F A B P 4$, and LPL mRNA expression decreased, and the ACC and FAS protein levels also decreased (Figure 1D,E; $p<0.05$ ).

\subsection{PLIN1 Affected Triglyceride Level and LD Sizes in Bovine Preadipocytes}

This study examinedLD and triglyceride content in adipocytes after overexpression of and interference with PLIN1. The number and size of LDs of fat cells were detected via oil red O staining. After overexpresion of PLIN1, the number of fat droplets in the fat cells increases and the volume becomes larger, promoting the accumulation of fat droplets in adipocytes. The number of lipid droplets decreased in bovine adipocytes after interference with PLIN1 (Figure 2A). The LDs were counted using ImageJ software. The relative counts of lipid droplets showed the same result in the staining pictures(Figure 2B; $p<0.05$ ). Upon overexpression of PLIN1, the triglyceide content in adipocytes was significantly higher than that in the control group, and the content of triglyceride in adipocytes after interference with PLIN1 was less than that in the control group(Figure 2 ; $p<0.05$ ). The results show that the PLIN1 gene plays crucial role in lipid metabolism. 


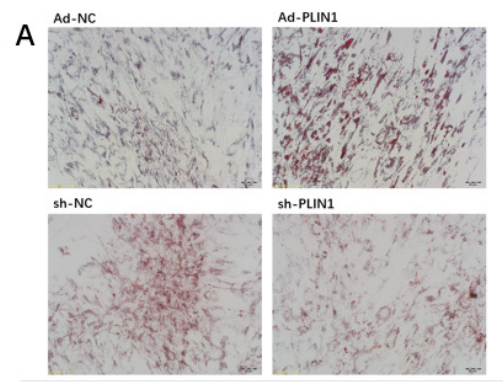

B

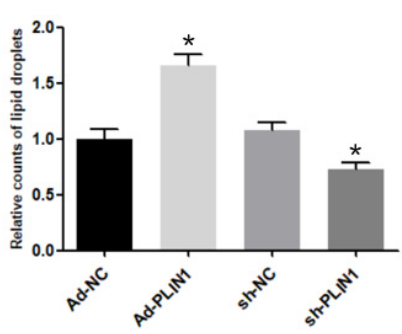

C
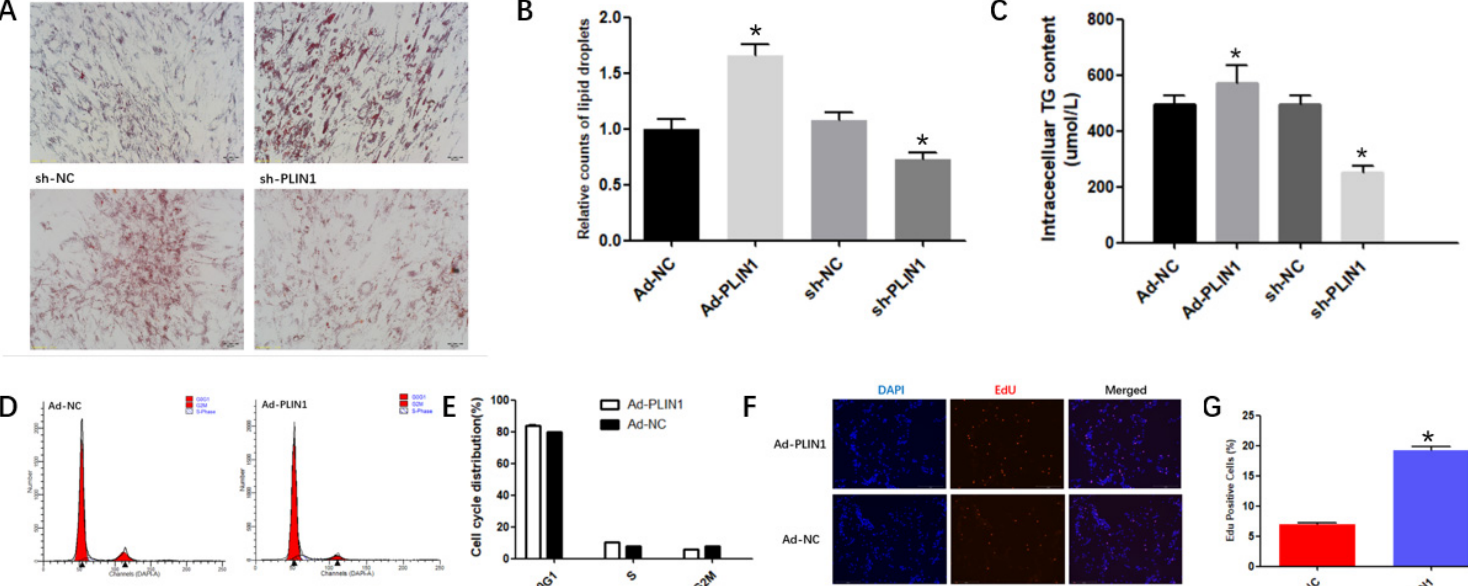

|
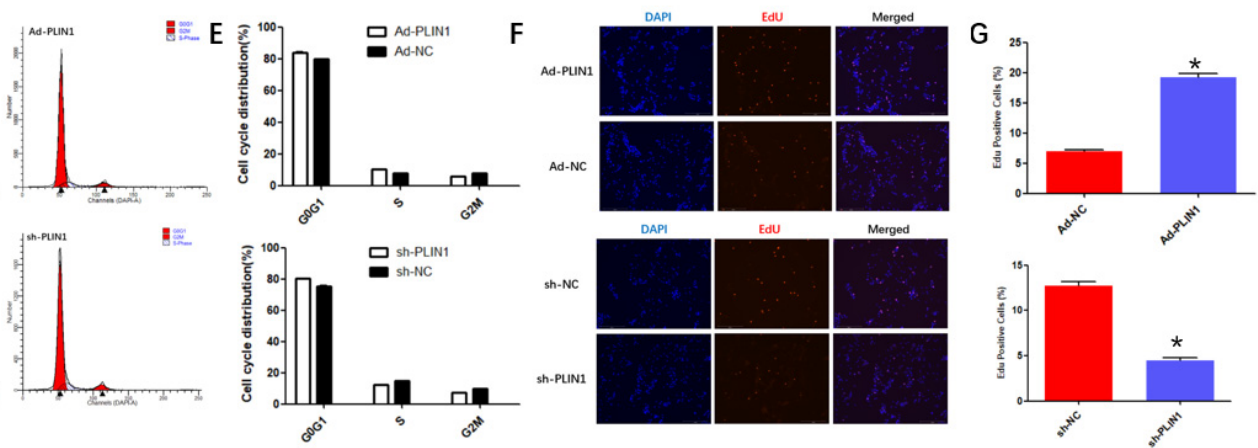

Figure 2. The PLIN1 gene affected triglyceride levels and LD formation in bovine adipocytes. (A,B) PLIN1 overexpression and interference observed by staining bovine adipocytes with oil red. The lipid droplets were counted using the ImageJ software. (C) Effects of overexpression and interference of the PLIN1 gene on triglyceride storage in bovine adipocytes (D,E) Flow cytometric analysis of PLIN1 gene in bovine adipocytes. $(\mathbf{F}, \mathrm{G})$ The cells are stained with EdU after four different adenovirus treatments. The cells were counted using the ImageJ software. The values represent mean $\pm \operatorname{SEM}(n=3) .{ }^{*} p<0.05$.

Furthermore, according to the results, interference with PLIN1 gene expression reduced the percentage of S-phase cells, while up-regulation of PLIN1 increased the percentage of S-phase cells (Figure 2D-F; $p<0.05$ ). To verify the effect of PLIN1 gene on the proliferation of bovine preadipocytes, EdU staining was used to investigate. Overexpression of PLIN1 increased the number of EdU-labeled cells. However, downregulation of PLIN1 significantly decreased the number of EdU-labelled cells (Figure 2G; $p<0.05$ ). In general, after interfering with PLIN1 gene, the mRNA expression of PLIN1 was reducing.

\subsection{Study of DEGs in Bovine Adipocytes}

In order to further study the mechanism of the influence of PLIN1 gene on the differentiation of bovine adipocytes, transcriptome sequencing experiments were carried out. After adding the adenovirus to the bovine adipocytes and culturing them in the differentiation medium for 6 days, cell samples were collected. The studied group was treated with Ad-PLIN1, whereas the control group was treated with Ad-NC. The limma or DESeq2 package was utilized to analyze the raw RNA-seq, and the obtained findings were then validated by RRA (Robust rank aggregation) with adjusted $p$-value $<0.05)$ using the $\log _{2}$ FC standard. Based on the RNA-seq results of the Ad-PLIN1 and Ad-NC treatment groups, a total of 1923 DEGs were detected, in which 765 genes were significantly upregulated and 1158 genes were significantly downregulated (Figure 3A; $p<0.05$ ). The genes with high expression showed as red color and genes with low expression were indicated by showing the blue genes (Figure 3B; $p<0.05$ ). The Ad-PLIN1 group and the control group (Ad-NC) jointly expressed 12336 genes, the overexpression group specifically expressed 557 genes, and the control group specifically expressed 429 genes (Figure 3C; $p<0.05$ ). 

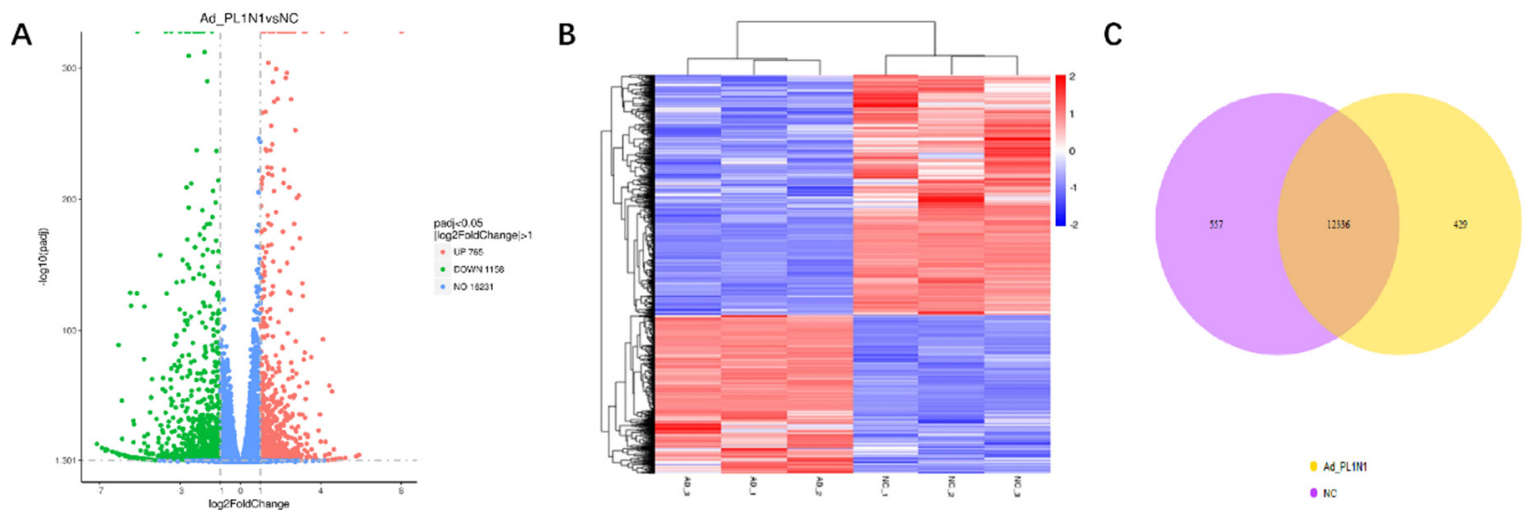

Figure 3. RNA-seq based DEGs between the groups. (A) The DEGs represented by volcanic map. The significant DEGs represented as upregulated (red dots) and downregulated (green dots), whereas, blue dots shows the non-significant differential expressed gens. (B) The DEGs presented by clustering map. The genes with high expression showed as Red color and genes with low expression were indicated by showing the blue genes. (C) The DEGs represented by Venn diagram. Venn diagram showed the overlapping portion by sharing the expression of pattern of the DEGs.

\subsection{GO Terms and KEGG Pathway Analysis of the DEGs}

In order to discover the relationship between the DEGs and phenotypic characteristics in adipocyte differentiation, the GO enrichment of DEGs was analyzed (Figure 4A). Among these genes, most were found to be involved in intracellular organelle homeostasis as well as cellular metabolic processes, indicating that different genes are coordinatly involved in biological processes. Furthermore, we classified the DEGs to reveal the enrichment of significant DEGs by using the KEGG database pathways. Based on the KEGG enrichment of DEGs, the majority of genes were found to play a crucial role in metabolic and cancer pathways (Figure 4B). Nonetheless, several DEGs were still associated with fatty acid biosynthesis, such as those of the PPAR, AMPK, and Wnt pathways (Figure 4C-E; $p<0.05)$. There were six differentially expressed genes in the AMPK signaling pathway in different treatment cells, two upregulated genes, $P P A R \gamma$ and $S C D 1$, and four down-regulated genes, $O b R b, F B P$, $P 13 K$, and $A M P K$ (Figure $4 C ; p<0.05$ ). There were eight differentially expressed genes in the PPAR signaling pathway in different treatment cells, and 6 up-regulated genes, namely HMGCS 2, ApoAI, PLTP, ACBP, PPAR $\gamma$ and SCD1; two downregulated genes, OLR1 and PGAR (Figure 4D; $p<0.05$ ). There were seven genes differentially expressed in the Wnt signaling pathway in different treatment cells, and four genes are up-regulated, namely RSPO, FRP, WNT11, and WNT 5; there were three down-regulated genes, namely GBP, NKD and DAAM1 (Figure 4E; $p<0.05$ ). 


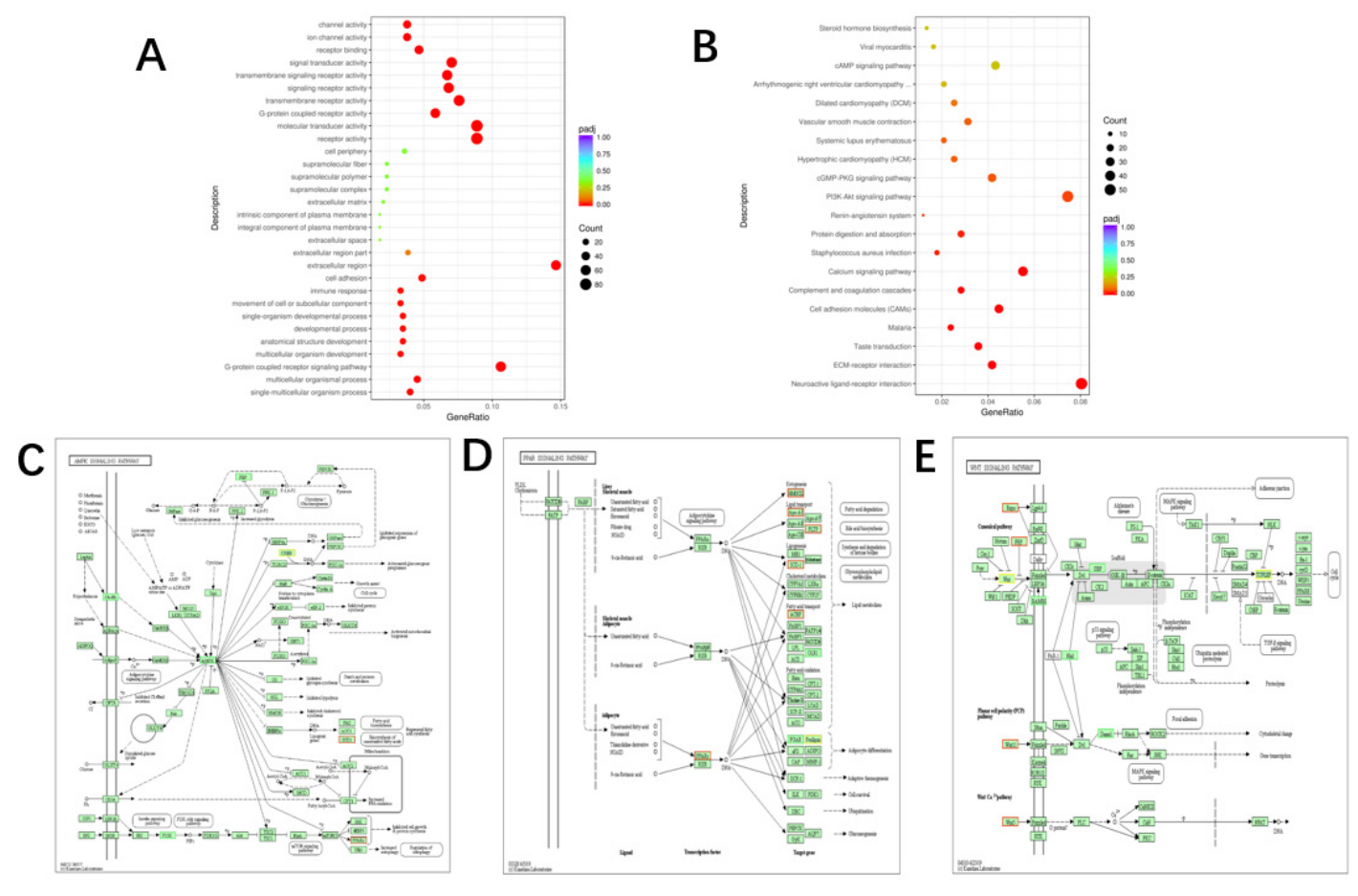

Figure 4. GO terms and KEGG pathway analysis of the DEGs Differentially Expressed Genes. (A) The differential expression of differential genes in the three GO terms of biological processes, cell components and molecular functions was sorted and analyzed. The 30 most significant GO terms are enriched. (B) The KEGG database was used to analyze the significance of pathway enrichment of differentially expressed genes, and the 20 pathways were significantly enriched. (C-E) Differentially expressed genes are enriched in signaling pathways such as AMPK signaling pathway, PPAR signaling pathway, and Wnt signaling pathway, which are related to fat metabolism.

\subsection{Identification of the DEGs during Promote of Differentiation in Bovine Adipocytes}

To validate the results of RNA-sequencing, fourteen DEGs were selected from the transcriptome sequencing results, and their expression in bovine adipocytes treated with different adenoviruses was verified by qRT-PCR with GAPDH as the internal reference gene. (Figure $5 ; p<0.05$ )

Table 2. Gene expression analysis, and differentially expressed genes Identification.

\begin{tabular}{cccc}
\hline Gene Name & Gene Full Name & $\begin{array}{c}\text { log2FoldChange } \\
\text { (Ad/NC) }\end{array}$ & $p$-Value \\
\hline ACSF2 & acyl-CoA synthetase family member 2 & 1.579670955 & $1.69 \mathrm{E}-221$ \\
RBP4 & retinol binding protein 4 & 0.775424841 & $6.02 \mathrm{E}-46$ \\
THBS3 & thrombospondin 3 & 0.828570053 & $3.32 \mathrm{E}-59$ \\
LUM & lumican & 0.720545336 & $1.99 \mathrm{E}-101$ \\
GLIPR2 & GLI pathogenesis related 2 & 0.845982741 & $1.59 \mathrm{E}-96$ \\
CSF1 & colony stimulating factor 1 & 1.252623086 & $1.33 \mathrm{E}-241$ \\
SYTL2 & synaptotagmin like 2 & 0.798285899 & $2.66 \mathrm{E}-132$ \\
NR4A1 & nuclear receptor subfamily 4 group A member 1 & 0.726639325 & $2.10 \mathrm{E}-33$ \\
PLTP & phospholipid transfer protein & 2.733646178 & $6.32 \mathrm{E}-256$ \\
PALLD & palladin, cytoskeletal associated protein & 0.852884845 & $2.09 \mathrm{E}-132$ \\
TMEM79 & transmembrane protein 79 & -0.864004155 & $6.77 \mathrm{E}-16$ \\
\hline CYR61 & cysteine rich angiogenic inducer 61 & 0.961861819 & $1.90 \mathrm{E}-154$ \\
ZFP36L1 & ZFP36 ring finger protein like 1 & -0.455224558 & $3.95 \mathrm{E}-29$ \\
SPOCK2 & SPARC/osteonectin, cwcr and kazal like domains & -1.811236856 & $9.02 \mathrm{E}-173$ \\
& proteoglycan 2 & & \\
\hline
\end{tabular}




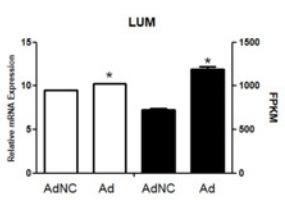

CSF1
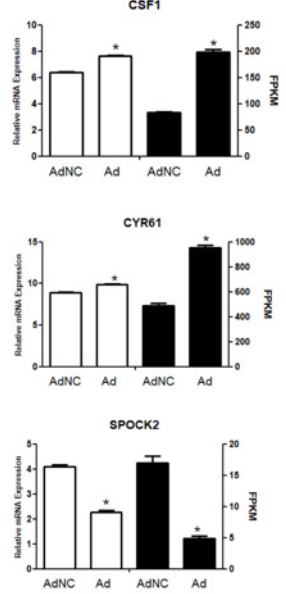

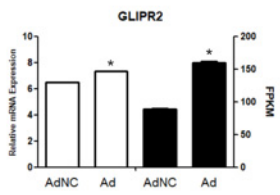

SYTL2
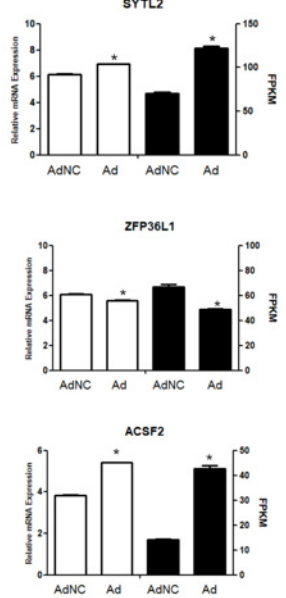
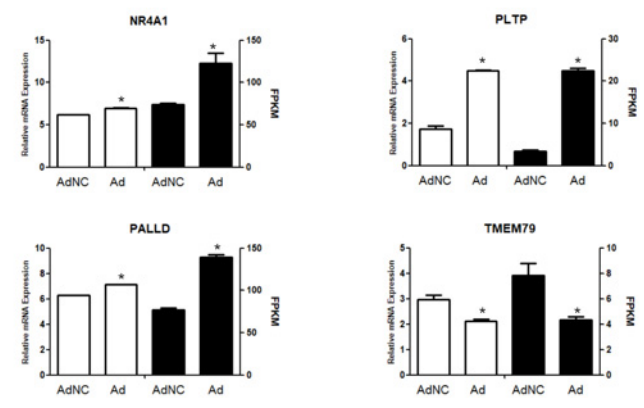

RBP4
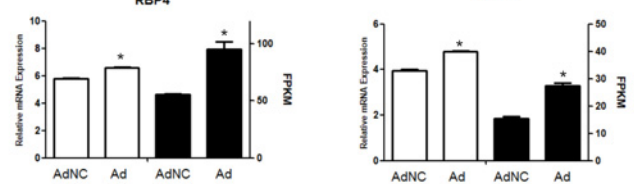

Figure 5. qPCR verification transcriptome sequencing of DEGs. Fourteen DEGs were selected, namely ACSF2, RBP4, THBS3, LUM, GLIPR2, CSF1, SYTL2, NR4A1, PLTP, PALLD, TMEM79, CYR61, ZFP36L1 and $S P O C K 2$, with GAPDH as the internal reference gene (Table 2).

\section{Discussion}

Adenovirus is a viral vector widely used in scientific research such as gene expression research and gene therapy. PLIN1 acts as a scaffold on the surface of LD and has a structural and regulatory role in the formation and function of LD [34]. Lipid droplets are dynamic organelles in the adipocytes. Under basic conditions, PLIN1 can prevent the breakdown of basic fat and increase lipid synthesis and LD formation [35]. Knocking down the PLIN1 gene increases basal lipolysis, reduces the size of LD in adipocytes, and leads to resistance to diet-induced obesity in mice [36].

In this study, triglyceride assay and oil red O staining showed increased expression of PLIN1, but PLIN1 silencing inhibited TAG synthesis and LD formation, consistent with previous studies in mice. The cell cycle can be divided into G1 (DNA synthesis preparation), S (DNA synthesis), S2 (division preparation), M phase (division), and G0 (quiescent) [37]. In the flow cytometry test, the cell DNA is stained with the fluorescent dye PI, and the DNA content in the cells was analyzed according to the fluorescence intensity, and then the percentage of the number of cells in G1/G0, S, and G2/M phases is determined [38]. Common methods for studying the cell cycle include BrdU, EdU, and CCK8 staining. DNA replication activity is detected based on the specific reaction between EdU and the fluorescent dye, and the proliferation of cells can be accurately reflected by detecting the EdU label.

In adipocytes, lipid droplets mainly store triglycerides and cholesterol esters, which hydrolyze when fatty acid levels are exhausted. HSL is an important enzyme involved in the hydrolysis of triglycerides (lipolysis) [39]. Studies have shown that HSL is highly expressed in adipocytes and has hydrolytic activity against triglycerides [40]. ATGL hydrolyzes triglycerides (TG) to diacylglycerol (DAG), while HSL hydrolyzes diacylglycerol DAG to monoacylglycerol (MAG). PLIN1 and PKA (protein kinase A) can directly or indirectly regulate the activity of HSL and ATGL [41]. PKA-mediated phosphorylation of HSL induces the docking of HSL with lipid droplet monolayers and then tightly binds with PLIN1 [42]. In this study, overexpression of PLIN1 significantly downregulate ATGL expression in terms of mRNA and protein, indicating that PLIN1 has an inhibitory effect on lipolysis at the level of gene transcription and protein translation.

The lipolysis processing of triglycerides in adipocytes is mainly governed by three factors: Lipase, lipid droplet coating proteins, and the lipolysis pathway. As the decisive factor for triglyceride 
hydrolysis, lipases mainly include adipocyte triglyceride lipase (ATGL), hormone-sensitive lipase (HSL) and monoacylglyceride lipase. The main marker genes in each stage of the fat differentiation process were selected to detect changes in their mRNA and protein expression levels. After overexpression of PLIN1, the mRNA expression levels of ACC, FASN, PPAR $\gamma, D G A T 2, F A B P 4$, and LPL were significantly increased, and the amount of FAS and PPAR $\gamma$ proteins also elevated. On the contrary, after interfering with the PLIN1 gene, ACC, FASN, PPAR $\gamma, D G A T 2, F A B P 4$, and LPL mRNA expression decreased, and ACC and FAS protein levels also decreased. Overexpression of PLIN1 suppresses the expression of ATGL mRNA, in contrast, it interferes with the PLIN1 gene, the mRNA and protein expression of ATGL rise.

The transcriptome is the sum of all RNA transcribed in the cell, including mRNA, non-coding RNA and small RNAs. Transcriptomics primarily involves studies of gene function at the RNA level, and plays an essential part in uncovering the molecular mechanisms of cells and tissues, as well as growth and development, and disease research [43]. Transcriptome sequencing technology is based on second-generation sequencing technology, which mainly analyzes gene expression levels, differential genes, new genes, and gene function annotations. It is an important means of gene function and gene expression. The development of second-generation sequencing technology has offered new approaches and approaches for functional genomics research [44]. RNA-seq has many advantages. It can detect known genes and predict new transcripts without designing probes and needing to know much information, with higher sensitivity and greater dynamic range [45].

The adipogenic differentiation of adipocytes is mainly divided into four stages: the first stage involves MSC differentiation into adipocytes, and the marker gene of this stage is LPL; in the second stage, adipocytes undergo growth inhibition with clonal proliferation, thereby forming precursor adipocytes. At this time the cells just begin to appear with fat droplets, and the marker genes are $A D D 1$ and $C / E B P \beta$; the third stage with respect to precursor adipocytes after growth, proliferation, and gene expression includes formation of immature adipocytes. At this time, the cells have a large number of small lipid droplets. The third stage marker genes are C / EBP $\alpha$ and $P P A R \gamma$.In relation to the deposition of fat in the cells, small lipid droplets converge into large lipid droplets to fill the fat cells, forming mature fat cells [46]. The fourth stage marker genes are GPD (glycerol triphosphate dehydrogenase), FAS (fatty acid synthase), ACC (acetyl coenzyme A decarboxylase), FABP4 (fatty fatty acid binding protein), PCK1 (Phosphoenolpyruvate Carboxykinase 1). With the transcriptome sequencing results, after overexpression of PLIN1, the expression levels of key genes FABP4, FAS, $D G A T 2$, and $A C C$ all increased during adipocyte differentiation. FABP4 is a fat-derived factor that is significantly upregulated during the differentiation of precursor adipocytes into adipocytes [47]. During the differentiation of precursor adipocytes, Hoxa5 inhibits the PKA/HSL signaling pathwayby regulating FABP4 and therefore promoting adipocyte differentiation and diminishing lipolysis [48].

The PAT family proteins PLIN1 and PLIN2 have the same and opposite regulatory effects on lipolysis in Drosophila, indicating the important role and complex functions of PAT family proteins during the lipolysis process [49]. In bovine adipocytes, the expression level of PLIN2 decreased and the expression level of PLIN3 gene increased after overexpression of PLIN1 gene according to RNA-Seq results. These findings indicate that PAT in bovine adipocytes is consistent with the studies in Drosophila family proteins, such that they not only have the same role in regulating lipid metabolism, but also exert complex opposite regulatory effects among PAT family proteins.

Exploring the genetic variation of candidate genes is an important method to promote Qinchuan beef cattle sub-marker breeding and crucial for economic correlation analysis. Our study shows that PLIN1 gene has a regulatory role in adipocyte differentiation and lipid metabolism, and a large number of new transcripts have also been detected in different processed adipocyte samples, which provides resources for further improvement of bovine genome annotation and molecular breeding research of beef cattle. 


\section{Conclusions}

In bovine adipocytes, overexpression of PLIN1 gene can promote the expression of fat metabolism-related genes at the mRNA and protein levels. Furthermore, overwxpression of PLIN1 shows that the number of lipid droplets in fat cells, their volume, and the content of triglyceride increased. The PLIN1 gene can promote the accumulation of triglycerides in bovine preadipocytes and has an important regulatory role in fat metabolism. RNA-seq was used to analyze differentially expressed genes in bovine preadipocytes after overexpression of adenoviruses Ad-PLIN1 and Ad-NC (empty virus). A total of 1923 differentially expressed genes were detected. The DEGs were enriched in members of the AMPK, Wnt, and PPAR signaling pathways related to fat proliferation and differentiation. This indicates that the PLIN1 gene has an important regulatory impact on fat proliferation and metabolism. Moreover, the sequencing results also screened new DEGs related to fat metabolism pathways, providing theoretical support for the molecular breeding of Qinchuan beef cattle.

Author Contributions: Conceptualization, S.L.; data curation, S.L., S.H.A.R. and C.Z.; formal analysis, S.L. and S.H.A.R.; funding acquisition, L.Z.; Methodology, S.L., S.H.A.R. and G.C.; project administration, L.Z.; Resources, L.Z.; Software, C.Z. and G.C.; supervision, L.Z.; validation, G.C.; writing-original draft, S.L. and S.H.A.R.; writing-review and editing, C.Z. All authors have read and agreed to the published version of the manuscript.

Funding: The present study was supported by grants from the National Science and Technology Support Projects (No. 2015BAD03B04), the National Beef Yak Industry Technology System (No. CARS-37), and the Major Agricultural Science and Technology Innovation and Transformation Plan in Shaanxi Province (No. NYKJ-2016-06).

Acknowledgments: We would like to thank Sayed Haidar Abbas Raza for critical reading of the manuscript.

Conflicts of Interest: The authors declare no conflict of interest.

\section{References}

1. Jeremiah, L.E. The influence of subcutaneous fat thickness and marbling on beef Palatability and consumer acceptability. Food Res. Int. 1996, 29, 513-520. [CrossRef]

2. Dolezal, H.G.; Smith, G.C.; Savell, J.W.; Carpenter, Z.L. Comparison of Subcutaneous Fat Thickness, Marbling and Quality Beef Grade for Predicting Palatability of beef. J. Food Sci. 1982, 47, 397-401. [CrossRef]

3. Martins, T.S.; Sanglard, L.M.; Silva, W.; Chizzotti, M.L.; Renno, L.N.; Serao, N.V.; Silva, F.F.; Guimaraes, S.E.; Ladeira, M.M.; Dodson, M.V.; et al. Molecular Factors Underlying the Deposition of Intramuscular Fat and Collagen in Skeletal Muscle of Nellore and Angus Cattle. PLoS ONE 2015, 10, e0139943. [CrossRef] [PubMed]

4. Raza, S.H.A.; Gui, L.; Khan, R.; Schreurs, N.M.; Xiaoyu, W.; Wu, S.; Mei, C.; Wang, L.; Ma, X.; Wei, D.; et al. Association between FASN gene polymorphisms ultrasound carcass traits and intramuscular fat in Qinchuan cattle. Gene 2018, 645, 55-59. [CrossRef] [PubMed]

5. Raza, S.H.A.; Shijun1, L.; Khan, R.; Schreurs, N.M.; Manzari, Z.; El-Aziz, A.H.A.; Ullah, I.; Kaster, N.; Shah, M.A.; Zan, L. Polymorphism of PLIN1 gene and their association with body measures and ultrasound carcass traits in Qinchuan beef cattle. Genome 2020, 63, 483-492. [CrossRef] [PubMed]

6. Cai, H.; Li, M.; Sun, X.; Plath, M.; Li, C.; Lan, X.; Lei, C.; Huang, Y.; Bai, Y.; Qi, X.; et al. Global Transcriptome Analysis During Adipogenic Differentiation and Involvement of Transthyretin Gene in Adipogenesis in Cattle. Front. Genet. 2018, 9, 463. [CrossRef]

7. Antoni, P.; Lawrence, C.; Perry, E.B. The PAT Family of Lipid Droplet Proteins in Heart and Vascular Cells. Curr. Hypertens. Rep. 2008, 10, 461-466.

8. Raza, S.H.A.; Khan, S.; Amjadi, M.; Abdelnour, S.A.; Ohran, H.; Alanazi, K.M.; Abd El-Hack, M.E.; Taha, A.E.; Khan, R.; Gong, C.; et al. Genome-wide association studies reveal novel loci associated with carcass and body measures in beef cattle. Arch. Biochem. Biophys. 2020, 13, 108543. [CrossRef]

9. Luo, J.; Deng, Z.-L.; Luo, X.; Tang, N.; Song, W.-X.; Chen, J.; Sharff, K.A.; Luu, H.H.; Haydon, R.C.; Kinzler, K.W. A protocol for rapid generation of recombinant adenoviruses using the AdEasy system. Nat. Protoc. 2007, 2, 1236-1247. [CrossRef]

10. He, T.-C.; Zhou, S.; Da Costa, L.T.; Yu, J.; Kinzler, K.W.; Vogelstein, B. A simplified system for generating recombinant adenoviruses. Proc. Natl. Acad. Sci. USA 1998, 95, 2509-2514. [CrossRef] 
11. Lee, B.; Zhu, J.; Wolins, N.E.; Cheng, J.X.; Buhman, K.K. Differential association of adipophilin and TIP47 proteins with cytoplasmic lipid droplets in mouse enterocytes during dietary fat absorption. Biochim. Biophys. Acta 2009, 1791, 1173-1180. [CrossRef]

12. Walther, T.C.; Farese, R.V., Jr. Lipid droplets and cellular lipid metabolism. Annu. Rev. Biochem. 2012, 81, 687-714. [CrossRef] [PubMed]

13. Sun, Z.; Gong, J.; Wu, H.; Xu, W.; Wu, L.; Xu, D.; Gao, J.; Wu, J.W.; Yang, H.; Yang, M.; et al. Perilipin1 promotes unilocular lipid droplet formation through the activation of Fsp27 in adipocytes. Nat. Commun. 2013, 4, 1594. [CrossRef] [PubMed]

14. Castro-Chavez, F.; Yechoor, V.K.; Wooten, E.C.; Sharma, S.; Saha, P.K.; O'Connell, P.; Martinez-Botas, J.; Taegtmeyer, H.; Chan, L. Coordinated Upregulation of Oxidative Pathways and Downregulation of Lipid Biosynthesis Underlie Obesity Resistance in Perilipin Knockout Mice. Diabetes 2003, 52, 2666-2674. [CrossRef]

15. Martinez-Botas, J.; Anderson, J.B.; Tessier, D.; Lapillonne, A.; Chang, B.H.-J.; Quast, M.J.; Gorenstein, D.; Chen, K.-H.; Chan, L. Absence of perilipin results in leanness and reverses obesity in Lepr db/db mice. Nat. Genet. 2000, 26, 474-479. [CrossRef]

16. Temprano, A.; Sembongi, H.; Han, G.-S.; Sebastián, D.; Capellades, J.; Moreno, C.; Guardiola, J.; Wabitsch, M.; Richart, C.; Yanes, O.; et al. Redundant roles of the phosphatidate phosphatase family in triacylglycerol synthesis in human adipocytes. Diabetologia 2016, 59, 1985-1994. [CrossRef]

17. Maurizi, G.; Poloni, A.; Mattiucci, D.; Santi, S.; Maurizi, A.; Izzi, V.; Giuliani, A.; Mancini, S.; Zingaretti, M.C.; Perugini, J.; et al. Human white adipocytes convert into "rainbow"adipocytes in vitro. J. Cell. Physiol. 2017, 232, 2887-2899. [CrossRef] [PubMed]

18. Zhai, W.; Xu, C.; Ling, Y.; Liu, S.; Deng, J.; Qi, Y.; Londos, C.; Xu, G. Increased Lipolysis in Adipose Tissues is Associated with Elevation of Systemic Free Fatty Acids and Insulin Resistance in Perilipin Null Mice. Horm. Metab. Res. 2010, 42, 247-253. [CrossRef]

19. Puri, V.; Ranjit, S.; Konda, S.; Nicoloro, S.M.; Straubhaar, J.; Chawla, A.; Chouinard, M.; Lin, C.; Burkart, A.; Corvera, S.; et al. Cidea is associated with lipid droplets and insulin sensitivity in humans. Proc. Natl. Acad. Sci. USA 2008, 105, 7833-7838. [CrossRef]

20. Lyu, Y.; Su, X.; Deng, J.; Liu, S.; Zou, L.; Zhao, X.; Wei, S.; Geng, B.; Xu, G. Defective differentiation of adipose precursor cells from lipodystrophic mice lacking perilipin 1. PLoS ONE 2015, 10, e0117536. [CrossRef]

21. Maurizi, G.; Petäistö, T.; Maurizi, A.; Della Guardia, L. Key-genes regulating the liposecretion process of mature adipocytes. J. Cell. Physiol. 2018, 233, 3784-3793. [CrossRef] [PubMed]

22. Gandotra, S.; Dour, C.L.; Bottomley, W.; Cervera, P.; Giral, P.; Reznik, Y.; Charpentier, G.; Auclair, M.; Delépine, M.; Barroso, I.; et al. Perilipin Deficiency and Autosomal Dominant Partial Lipodystrophy. N. Engl. J. Med. 2011, 364, 740-748. [CrossRef] [PubMed]

23. Rodrigues, M.; Griffith, L.G.; Wells, A. Growth factor regulation of proliferation and survival of multipotential stromal cells. Stem Cell Res. Ther. 2010, 1, 32. [CrossRef] [PubMed]

24. Shijun, L.; Khan, R.; Raza, S.H.A.; Jieyun, H.; Chugang, M.; Kaster, N.; Gong, C.; Chunping, Z.; Schreurs, N.M.; Linsen, Z. Function and characterization of the promoter region of perilipin 1 (PLIN1): Roles of E2F1, PLAG1, C/EBP $\beta$, and SMAD3 in bovine adipocytes. Genomics 2020, 112, 2400-2409. [CrossRef] [PubMed]

25. Hong, J.; Li, S.; Wang, X.; Mei, C.; Zan, L. Study of expression analysis of SIRT4 and the coordinate regulation of bovine adipocyte differentiation by SIRT4 and its transcription factors. Biosci. Rep. 2018, 38. [CrossRef] [PubMed]

26. Schmittgen, T.D. Real-Time Quantitative PCR. Methods 2001, 25, 383-385. [CrossRef]

27. Zongqian, N.; Zhiqi, S.; Luxin, Y.; Yon, T.S.; Jianli, S.; Peng, L. Fat-specific protein 27 undergoes ubiquitindependent degradation regulated by triacylglycerol synthesis and lipid droplet formation. J. Biol. Chem. 2010, 285, 9604-9615.

28. Cao, Y.; Toh, S.Y.; Gong, J.; Du, G.; Li, J.Z.; Yang, S.; Ye, J.; Yao, H.; Zhang, Y.; Xue, B.; et al. Up-Regulation of Mitochondrial Activity and Acquirement of Brown Adipose Tissue-Like Property in the White Adipose Tissue of Fsp27 Deficient Mice. PLoS ONE 2008, 3, e2890.

29. Da, W.H.; Sherman, B.T.; Lempicki, R.A. Systematic and integrative analysis of large gene lists using DAVID bioinformatics resources. Nat. Protoc. 2008, 4, 44-57.

30. Huang, D.W.; Sherman, B.T.; Lempicki, R.A. Bioinformatics enrichment tools: Paths toward the comprehensive functional analysis of large gene lists. Nucleic Acids Res. 2009, 37, 1-13. [CrossRef] 
31. Xie, C.; Mao, X.; Huang, J.; Ding, Y.; Wu, J.; Dong, S.; Kong, L.; Gao, G.; Li, C.Y.; Wei, L. KOBAS 2.0: A web server for annotation and identification of enriched pathways and diseases. Nucleic Acids Res. 2011, 39 (Suppl. S2), W316-W322. [CrossRef] [PubMed]

32. Wu, J.; Mao, X.; Cai, T.; Luo, J.; Wei, L. KOBAS server: A web-based platform for automated annotation and pathway identification. Nucleic Acids Res. 2006, 34, W720-W724. [CrossRef] [PubMed]

33. Livak, K.J.; Schmittgen, T.D. Analysis of relative gene expression data using real-time quantitative PCR and the 2(-Delta Delta C(T)) Method. Methods 2001, 25, 402. [CrossRef] [PubMed]

34. Ikura, Y.; Caldwell, S.H. Lipid droplet-associated proteins in alcoholic liver disease: A potential linkage with hepatocellular damage. Int. J. Clin. Exp. Pathol. 2015, 8, 8699-8708.

35. Beate Katharina, S.; Pamela, S.; Hans, H.; Ralf, Z.; Peter, S. Differential pattern of lipid droplet-associated proteins and de novo perilipin expression in hepatocyte steatogenesis. Hepatology 2010, 47, 1936-1946.

36. Krahmer, N.; Hilger, M.; Kory, N.; Wilfling, F.; Stoehr, G.; Mann, M.; Farese, R.V.; Walther, T.C. Protein Correlation Profiles Identify Lipid Droplet Proteins with High Confidence. Mol. Cell. Proteom. 2013, 12, 1115-1126. [CrossRef]

37. Merkel, D.E.; Mcguire, W.L. Ploidy, proliferative activity and prognosis. DNA flow cytometry of solid tumors. Cancer 1990, 65, 1194-1205. [CrossRef]

38. Corvò, R.; Giaretti, W.; Sanguineti, G.; Geido, E.; Barbieri, M. In vivo cell kinetics in head and neck squamous cell carcinoma predicts local control and helps guide radiotherapy regimen. J. Clin. Oncol. Off. J. Am. Soc. Clin. Oncol. 1995, 13, 1843-1850. [CrossRef]

39. Holm, C.; Kirchgessner, T.; Svenson, K.; Fredrikson, G.; Nilsson, S.; Miller, C.; Shively, J.; Heinzmann, C.; Sparkes, R.; Mohandas, T. Hormone-sensitive lipase: Sequence, expression, and chromosomal localization to 19 cent-q13.3. Science 1988, 241, 1503-1506. [CrossRef]

40. Xia, B.; Cai, G.H.; Yang, H.; Wang, S.P.; Wu, J.W. Adipose Tissue Deficiency of Hormone-Sensitive Lipase Causes Fatty Liver in Mice. PLoS Genet. 2017, 13, e1007110. [CrossRef]

41. Sztalryd, C. Perilipin A is essential for the translocation of hormone-sensitive lipase during lipolytic activation. J. Cell Biol. 2003, 161, 1093-1103. [CrossRef] [PubMed]

42. Granneman, J.G.; Moore, H.P.H.; Granneman, R.L.; Greenberg, A.S.; Obin, M.S.; Zhu, Z. Analysis of Lipolytic Protein Trafficking and Interactions in Adipocytes. J. Biol. Chem. 2007, 282, 5726-5735. [CrossRef] [PubMed]

43. Wang, Z.; Gerstein, M.; Snyder, M. RNA-Seq: A revolutionary tool for transcriptomics. Nat. Rev. Genet. 2009, 10, 57-63. [CrossRef] [PubMed]

44. Shendure, J.; Ji, H. Next-generation DNA sequencing. Nat. Biotechnol. 2008, 26, 1135-1145. [CrossRef]

45. Croucher, N.J.; Thomson, N.R. Studying bacterial transcriptomes using RNA-seq. Curr. Opin. Microbiol. 2010, 13, 619-624. [CrossRef]

46. Park, Y.; Storkson, J.M.; Ntambi, J.M.; Cook, M.E.; Sih, C.J.; Pariza, M.W. Inhibition of hepatic stearoyl-CoA desaturase activity by trans -10 , cis -12 conjugated linoleic acid and its derivatives. BBA Mol. Cell Biol. Lipids 2000, 1486, 285-292. [CrossRef]

47. Hotamisligil, G.S.; Bernlohr, D.A. Metabolic functions of FABPs-mechanisms and therapeutic implications. Nat. Rev. Endocrinol. 2015, 11, 592. [CrossRef]

48. Cao, W.; Xu, Y.; Luo, D.; Saeed, M.; Sun, C. Hoxa5 Promotes Adipose Differentiation via Increasing DNA Methylation Level and Inhibiting PKA/HSL Signal Pathway in Mice. Cell. Physiol. Biochem. 2018, 45, 1023-1033. [CrossRef]

49. Bi, J.; Xiang, Y.; Chen, H.; Liu, Z.; Gronke, S.; Kuhnlein, R.P.; Huang, X. Opposite and redundant roles of the two Drosophila perilipins in lipid mobilization. J. Cell Sci. 2012, 125, 3568-3577. [CrossRef]

Publisher's Note: MDPI stays neutral with regard to jurisdictional claims in published maps and institutional affiliations.

(C) 2020 by the authors. Licensee MDPI, Basel, Switzerland. This article is an open access article distributed under the terms and conditions of the Creative Commons Attribution (CC BY) license (http://creativecommons.org/licenses/by/4.0/). 